\title{
The role of DNA integrity in opportunistic longitudinal telomere studies
}

\author{
Malin Undin ${ }^{1}$, Kristene Gedey ${ }^{2}$, Simon Hills $^{1}$, and Isabel Castro ${ }^{2}$ \\ ${ }^{1}$ Massey University College of Sciences \\ ${ }^{2}$ Massey University
}

October 23, 2020

\begin{abstract}
Determining both individual age and population age distribution is crucial for an array of ecological studies. This is generating growing interest in molecular age markers such as telomere length. Most studies exploring the relationship between age and telomere length have been cross-sectional, but such studies face problems with large individual variation and the selective loss paradox. Thus, interest is growing rapidly for turning to longitudinal studies. In this study, the rate of telomere loss was analyzed for the extraordinarily long-lived North Island brown kiwi, Apteryx mantelli. Relative telomere length (RTL) was quantified using qPCR from blood from four separate sampling occasions across 14 years were analysed. Uniquely, the analysis of RTL was combined with high resolution analysis of genomic quality to get numerical values of DNA integrity. The analysis of RTL suggested a circa $5 \%$ annual increase in A. mantelli telomere length. However, RTL was found to be highly correlative with DNA integrity, indicating that the perceived elongation of telomeres was a result of DNA quality differences between cohorts. Notably, the observed, positive correlation remained significant even when analyzing only samples classified as being of high DNA quality. Previous work has highlighted the potential impact of sample storage differences on RTL. However, to our knowledge, this is the first study to suggest that even small differences in DNA integrity between samples cohorts can impact the results of telomere studies. These findings are of great importance since longitudinal telomere studies of long-lived species tend to be "after the fact" utilizing already available samples for which handling and/or storage regimes might differ or be unknown. For such studies, we suggest that analysis of DNA quality with higher precision than traditional gel electrophoresis is needed to generate reliable results of telomere dynamics.
\end{abstract}

\section{Hosted file}

Main Document_The role of DNA integrity in opportunistic longitudinal telomere studies_220ct2020.pdf available at https://authorea.com/users/369595/articles/488424-the-role-of-dna-integrityin-opportunistic-longitudinal-telomere-studies 